\title{
On the structure of giant HII regions and HII galaxies
}

\author{
G. Tenorio-Tagle ${ }^{1}$, \\ C. Muñoz-Tuñón ${ }^{2}$, E. Pérez ${ }^{3}$, S. Silich ${ }^{1}$ and E. Telles ${ }^{4}$ \\ ${ }^{1}$ Instituto Nacional de Astrofísica Optica y Electrónica, AP 51, 72000 Puebla, México \\ email: gtt@inaoep.mx \\ ${ }^{2}$ Instituto de Astrofísica de Canarias, E 38200 La Laguna, Tenerife, Spain \\ cmt@ll.iac.es \\ ${ }^{3}$ Instituto de Astrofísica de Andalucía (CSIC), Camino bajo de Huetor 50, E 18080 Granada, \\ Spain \\ eperez@iaa.es \\ ${ }^{4}$ Observatório Nacional, Rua José Cristino 77, 20921-400, Rio de Janeiro, Brazil \\ etelles@on.br
}

\begin{abstract}
We review the structural properties of giant extragalactic HII regions and HII galaxies based on two dimensional hydrodynamic calculations, and propose an evolutionary sequence that accounts for their observed detailed structure. The model assumes a massive and young stellar cluster surrounded by a large collection of clouds. These are thus exposed to the most important star-formation feedback mechanisms: photoionization and the cluster wind. The models show how the two feedback mechanisms compete with each other in the disruption of clouds and lead to two different hydrodynamic solutions: The storage of clouds into a long lasting ragged shell that inhibits the expansion of the thermalized wind, and the steady filtering of the shocked wind gas through channels carved within the cloud stratum that results into the creation of large-scale superbubbles. Both solutions are here claimed to be concurrently at work in giant HII regions and HII galaxies, causing their detailed inner structure.
\end{abstract}

Keywords. galaxies: starburst - galaxies: galaxies: HII - galaxies: ISM: HII regions

\section{Introduction}

Multiple studies during the last decades have addressed the impact of photoionization, stellar winds and supernova explosions on interstellar matter (ISM). Disruptive events restructure the birth place of massive stars and their surroundings, while leading to multiple phase transitions in the ISM (see e.g. Comeron 1997; Yorke et al. 1989; GarcíaSegura et al. 2004; Hosokawa \& Inutsuka 2005 and Tenorio-Tagle \& Bodenheimer 1988 and references therein). On the other hand, giant molecular clouds, the sites of ongoing star formation, present a hierarchy of clumps and filaments of different scales whose volume filling factor varies between $10 \%$ to $0.1 \%$ (see McLow \& Klessen, 2004, and references therein). As the efficiency of star formation in molecular clouds is estimated to be $\leqslant 10 \%$ (Larson 1988; Franco et al. 1994) the implication is that the bulk of the cloud structure remains after a star forming episode. All of these studies are relevant within the fields of interstellar matter and star formation and, in particular, regarding the physics of feedback, a major ingredient in the evolution of galaxies and thus in cosmology.

From the observations, we know that giant extragalactic HII regions and HII galaxies are excellent examples of the impact of massive stars on the ISM. They belong to the same class of objects because they are powered by young massive bursts of star formation, and 
because of their similar physical size, morphology and inner structure. Detailed studies of 30 Doradus (see Chu \& Kennicutt 1994; Melnick et al. 1999), NGC 604 (Sabalisck et al. 1995; Yang et al.1996) and several giant extragalactic HII regions and HII galaxies (Muñoz-Tuñón et al. 1996; Telles et al. 2001; Maíz-Apellániz et al. 1999) have been designed with the aim of unveiling the inner structure and dynamics of the nearest examples. All of them present a collection of nested shells that enclose an X-ray emitting gas and that may extend up to kpc scales. Some of the largest shells have stalled while others present expansion speeds of up to several tens of $\mathrm{km} \mathrm{s}^{-1}$. Detailed HST images have also confirmed these issues for HII galaxies. All of these sources, as in the studies of Telles et al. (2001) and Cairós et al. (2001), present a central bright condensation coincident with the massive burst of stellar formation. In giant HII regions and in some HII galaxies, this is resolved as the brightest filament or a broken ragged shell sitting very close to the exciting cluster.

\section{Feedback from massive star clusters}

The energy powering these giant volumes comes from the recently found unit of massive star formation: super star clusters (Ho 1997). Massive concentrations of young stars within the range of $10^{5} \mathrm{M}_{\odot}$ to several $10^{6} \mathrm{M}_{\odot}$ are all within a small volume $\sim 3-10 \mathrm{pc}$. The mechanical energy and the UV photon output from a massive stellar cluster is here confronted with an ISM structured into a collection of clouds. A full description of the following calculations can be found in Tenorio-Tagle et al. (2006). Animated versions of the models presented can be found at http://www.iaa.csic.es/ ${ }^{\sim}$ eperez/ssc/ssc.html.

There are two competing events: The pressure acquired by the wind at the reverse shock $\left(P_{b u b b l e}=\rho_{w} v_{\infty}^{2}\right)$ defines the velocity that the leading shock may have as it propagates into the intercloud medium $\left(V_{S}=\left(P_{b u b b l e} / \rho_{i c}\right)^{0.5}\right)$, and thus, as a first approximation, if the cloudlet distribution has an extent $D_{c l}$, then the time for the leading shock to travel across it is $t_{s}\left(=D_{c l} / V_{S}\right)$. On the other hand, the pressure gradient between ionized cloudlets and the intercloud medium, established through photoionization, is to disrupt clouds and lead to a constant density medium in a time $t_{d}=\alpha d_{c s} /\left(2 c_{H I I}\right)$; where $d_{c s} / 2$ is half the average distance or separation between clouds, $c_{H I I}$ is the sound velocity in the ionized medium and $\alpha$ is a small number $(\sim 4-6)$ and accounts for the number of times that a rarefaction wave ought to travel (at the sound speed) the distance $d_{c s} / 2$ to replenish the whole volume with an average even density $\langle\rho\rangle$. In this way if the cloud disruption process promoted by photoionization leads to an average $\langle\rho\rangle$ that could largely reduce the velocity of the leading shock $\left(V_{S}=\left(P_{\text {bubble }} /\langle\rho\rangle\right)^{0.5}\right)$, then $t_{s}$ could be larger than $t_{d}$, leading, as shown below, to an effective confinement of the shocked wind, at least for a significant part of the evolution.

Cases considering both feedback events lead to a rapid evolution of the photoionized cloud stratum, filling almost everywhere the low density intercloud zones, while spreading the density of the outermost cloudlets into the surrounding intercloud medium. This causes the development of an increasingly larger ionized expanding rim around the cloudlet distribution and leads to a rapid enhancement of the intercloud density. On the other hand, the wind - cloudlet interaction leads to a global reverse shock into the wind, that evolves from an initially ragged surface across which the isotropic wind is only partly thermalized, to an almost hemispherical surface that fully thermalizes the wind. Initially, after crossing the reverse shock the hot wind drives the leading shock into the cloudlet distribution and this immediately looks for all possible paths of least resistance in between clouds. This fact, diverts the shocked wind into multiple streams behind every overtaken cloudlet, diminishing steadily its power to reach the end of the 
cloudlet distribution before the outermost clouds expand and block many of the possible exits into the low density background gas.

In the calculation only one channel, the initially widest channel close to the grid equatorial axis, across which the original cloudlet spacing was set slightly larger than in the rest of the distribution, is successfully crossed by the leading shock and the thermalized wind behind it, after $\sim 10^{5}$ yr of evolution. A second channel through the cloud stratum is completed (close to the symmetry axis) after a time $t=5 \times 10^{5} \mathrm{yr}$. All other possibilities, are blocked by the large densities resultant from the champagne bath.

\section{Discussion}

We have shown here the effects of feedback from a massive stellar cluster into a selected cloudlet distribution, which although arbitrary, as it could have had a different extent or it could have considered clouds of different sizes, separations and locations, it has allowed us to explore a wide range of possibilities. These however, have lead to the two main possible physical solutions to be expected: partial pressure confinement of the shocked wind, and a stable and long-lasting filtering of the thermalized wind through the cloudlet stratum. Thus despite the arbitrary and simple boundary and initial conditions here assumed, the structures that develop within the flow resemble the structure of giant HII regions and HII galaxies. In particular we refer to the giant and multiple well structured shells evident at optical wavelengths, often referred to as nested shells (see Chu \& Kennicutt 1994), that enclose a hot X-ray emitting gas, as in 30 Dor (Wang 1999) and NGC 604 (Maíz-Apellániz et al. 2004). The calculations also lead to the slowly expanding brightest filament (or ragged shell) present in all sources close and around the exciting stars, and to the elongated, although much fainter, filaments on either side of the open channels that reassemble the ends of elongated columns into the giant superbubbles.

None of these structural features appear in calculations that assume a constant density ISM, nor in those that allow for a constant density molecular cloud as birth place of the exciting sources, or in calculations where the sources are embedded in a plane stratified background atmosphere. For all of these features to appear, a clumpy circumstellar medium seems to be a necessary requirement. A medium that would refrain the wind from an immediate exit into the surrounding gas and that would also allow for the build up of multiple channels through which the wind energy would flow in a less unimpeded manner. And thus giant HII regions and HII galaxies, both powered by massive star formation events producing an ample supply of UV photons and a powerful wind mechanical energy, all of them seem to process their energy into a stratum of dense cloudlets sitting in the immediate vicinity of the star formation event and this leads to the repeated structure common to all giant nebulae.

\section{Acknowledgements}

This study has been partly supported by grants AYA2004-08260-C03-01 and AYA 2004-02703 from the Spanish Ministerio de Educación y Ciencia, grant TIC-114 from Junta de Andalucía and Conacyt (México) grant 47534-F.

\section{References}

Cairós, L. M., Caon, N., Vílchez, J. M., González-Pérez, J. N. \& Muñoz-Tuñón, C. 2001, ApJS 136,393

Chu, Y. H. \& Kennicutt, R. 1994, ApJ 425, 720

Comeron, F. 1997, A\&GA 326, 1195 
Franco, J., Shore, S. \& Tenorio-Tagle, G. 1994, ApJ 436,795

Garcia-Segura, G. \& Franco, J. 2004, RMxAC 22,131

Ho, L. C. 1997, Rev.Mex.AA, Conf. Ser. 6, 5

Hosokawa, T. \& Inutsuka, S. 2005, astro-ph/0511165

Larson, R. 1988, in: R. E. Pudritz \& J. M. Fich (eds.), Galactic and Extra Galactic Star Formation (Dordrecht: Kluwer), p. 459

Mac Low, M.-M. \& Klessen, R. S. 2004, Rev. Mod. Phys. 76, N1, 125

Maíz-Apellániz, J., Muñoz-Tuñón, C., Tenorio-Tagle, G., Mas-Hesse, J. M. 1999, A\&̇A 343, 64

Melnick, J., Tenorio-Tagle, G. \& Terlevich, R. 1999, MNRAS 302, 677

Muñoz-Tuñón, C., Tenorio-Tagle, G., Castaneda, H. O. \& Terlevich, R. 1996, AJ 112, 1636

Sabalisck, N. S. P., Tenorio-Tagle, G., Castaneda, H. O. \& Muñoz-Tuñón, C. 1995, ApJ 444, 200

Telles, E., Muñoz Tuñón, C. \& Tenorio-Tagle, G. 2001, ApJ 548, 671

Tenorio-Tagle, G. \& Bodenheimer, P. 1988, ARA\&A 26, 145

Tenorio-Tagle, G. Silich, S. \& Muñoz-Tuñón, C. 2005, in: D. Vals-Gabaud \& M. Chavez (eds.), Resolved Stellar Populations (APS-CS), in press

Tenorio-Tagle, G., Muñoz Tuñón, C., Pérez, E., Silich, S. \& Telles, E. 2006, ApJ 643, 186

Yang, H., Chu, Y., Skillman, E. D. \& Terlevich, R. 1996, AJ 112, 146

Yorke, H. W., Tenorio-Tagle, G., Bodenheimer, P. \& Rozyczka, M. 1989, A\&̋A 216, 207

\section{Discussion}

B. Elmegreen: What kind of coherent resistance to the wind will an interconnecting magnetic field give if the field connects the clouds with the intercloud medium?

G. Tenorio-TAgle: A large magnetic pressure would enhance the resistance that the ragged shell presents to the shocked wind. In such cases I expect the ragged shell to have a longer life time despite its nearness to the exciting sources.

S. SHORE: Your calculational scenario has in effect created a "fluffy screen" so your flow is more or less a supersonic grid turbulence (without the ionization effects) so: what is the effective Reynolds number for the large scale? How do your results change with changes in the resolution? Have you tried to use a random and/or fractal structure for the surrounding medium? You might compare your results with laboratory studies.

G. Tenorio-Tagle: I am sorry but I don't understand what do you mean by a "fluffy screen". We have included in our calculations a realistic computational representation of the feedback from super stellar clusters. Many test calculations reassure us that the results are independent of the adopted numerical resolution. We would like indeed to follow your suggestion and surround our clusters by a random fractal structure. However, I would expect that if a significant degree of clumpiness is adopted as initial condition, then the two solutions here described would be recovered.

J. TURNeR: Have you included the effects of gravity in these models? For SSCs, this could be an important effect, and could, like in stars, facilitate a smooth transition to supersonic flow.

G. Tenorio-Tagle: Gravity is not relevant to super stellar clusters able to drive a stationary wind. This is because of the efficient thermalization of the deposited matter which leads to large temperatures $\left(T \sim 10^{7} \mathrm{~K}\right)$ and thus to sound velocities that well exceed the escape velocity. 